\title{
Real-time ultrasound-guided spinal anesthesia for cesarean section in patient with severe kyphoscoliosis and Duchenne's muscular dystrophy - A case report -
}

Received March 7, 2018

Revised 1st, May 5, 2018

2nd, July 5, 2018

3rd, July 5, 2018

Accepted August 11, 2018

\author{
Corresponding author \\ Hyungtae Kim, M.D. \\ Department of Anesthesiology \\ and Pain Medicine, Asan Medical \\ Center, University of Ulsan College \\ of Medicine, 88 Olympic-ro 43-gil, \\ Songpa-gu, Seoul 05505, Korea \\ Tel: 82-2-3010-3868 \\ Fax: 82-2-6008-2475 \\ E-mail: ingwei2475@naver.com \\ ORCID \\ http://orcid.org/0000-0003-2488-9986
}

\section{Hyungtae $\mathrm{Kim}^{1}$ and Sung In Shin ${ }^{2}$}

Department of Anesthesiology and Pain Medicine, ${ }^{1}$ Asan Medical Center, University of Ulsan College of Medicine, Seoul, ${ }^{2}$ Presbyterian Medical Center, Jeonju, Korea

Most elective cesarean sections are conducted under spinal anesthesia. Regional anesthesia has become the preferred technique, because general anesthesia is associated with a greater risk of maternal morbidity and mortality. In patients without absolute contraindication, spinal anesthesia is avoided, when procedural difficulty is increased by severe spinal deformity. A 41-year-old female patient was 33 weeks into pregnancy. Her height and weight were $145 \mathrm{~cm}$ and $45 \mathrm{~kg}$. The patient was planned for emergency cesarean section, due to cephalopelvic disproportion. Spinal anesthesia was planned since she was suffering from Duchenne's muscular dystrophy, and had risks of respiratory failure and prolonged mechanical ventilation after general anesthesia. However, the patient also had severe kyphoscoliosis, maybe due to Duchenne's muscular dystrophy. We are reporting a successful spinal anesthesia using real-time ultrasound guidance, for cesarean section in this obstetric patient with severe kyphoscoliosis.

Keywords: Cesarean section; Muscular dystrophy; Scoliosis; Spinal anesthesia; Ultrasonography.

Most elective cesarean sections are conducted under spinal anesthesia. Regional anesthesia has become the preferred technique because general anesthesia is associated with a greater risk of maternal morbidity and mortality [1]. However, there are limitations when administering spinal anesthesia in patients with spinal deformity.

Recent advancement and spread of ultrasound, allowed us to check the structures of spine and its surroundings. Preprocedural ultrasound evaluation of spine structure is beneficial, in facilitating central neuraxial block in patients with indistinct or impalpable surface, bony landmarks, or abnormal spinal anatomy [2-6], as it delineates the anatomy and allows for a more accurate estimation of the appropriate site and trajectory of needle insertion.

We are reporting a successful spinal anesthesia using realtime ultrasound guidance, for cesarean section in a patient with a severe kyphoscoliosis and Duchenne's muscular dystrophy.

\section{CASE REPORT}

A 41-year-old female patient was 33 weeks into pregnancy. Her height and weight were $145 \mathrm{~cm}$ and $45 \mathrm{~kg}$. The patient was planned for emergency cesarean section due to cephalopelvic disproportion. Cesarean section under spinal anesthesia was planned since she was suffering from Duchenne's

This is an Open Access article distributed under the terms of the Creative Commons Attribution Non-Commercial License (http://creativecommons.org/licenses/by-nc/4.0) which permits unrestricted non-commercial use, distribution, and reproduction in any medium, provided the original work is properly cited. 
muscular dystrophy and had risks of respiratory failure and prolonged mechanical ventilation after general anesthesia. However, the patient also had severe thoracolumbar kyphoscoliosis maybe due to Duchenne's muscular dystrophy (Fig. 1), therefore an informed consent was received to conduct the surgery under general anesthesia if ultrasound-guided spinal anesthesia failed.

Premedication before anesthesia was not administered. After arriving at the operating room, electrocardiography, noninvasive blood pressure monitor and pulse oximeter were attached. The patient's surface landmarks were poorly palpable, because the lumbar spines were severely rotated (about 115 degree) by marked scoliosis. Pre-procedural ultrasound scan and marking of insertion site were conducted, using ultrasound with the patient in a right lateral position (Fig. 1). Transportable ultrasound equipment with a $60 \mathrm{~mm}$ convex 2-5 MHz transducer (SonoSite M-Turbo ${ }^{\mathrm{TM}}$, USA) was used. Due to severely rotated lumbar spines, the spinous process was not palpable. Therefore, we confirmed spinous process and lamina by using ultrasound. Then images of interlaminar space and intrathecal space were produced by rotating and angulating the transducer, from cephalad-to-caudad and lateral-to-medial direction (Fig. 2).

After determining the insertion site, real-time ultrasoundguided spinal anesthesia was conductedwith the patient in a right lateral position. The skin was disinfected. Ultrasound transducer and cable were protected with a sterile ultrasound probe cover (Sono $\mathrm{Lab}^{\mathrm{TM}}, 18 \times 120 \mathrm{~cm}$, Korea). Then the needle (Spinocan ${ }^{\mathrm{TM}}, 0.53 \times 88 \mathrm{~mm}$, Germany) was carefully inserted in a paramedian approach, by real-time ultrasound- guided in-plane technique, and dural puncture was successfully achieved on the first attempt (Supplementary Video 1). To prevent spinal root and blood vessel puncture, the patient's symptoms and blood aspiration were monitored, while administering the spinal anesthesia by checking ultrasound image and needle position in real-time. After checking the cerebrospinal fluid, $9 \mathrm{mg}$ of $0.5 \%$ bupivacaine and $0.1 \mathrm{mg}$ of morphine sulfate were carefully injected. The patient was moved into a supine position. Anesthesia level was approximately T5 dermatome and the operation ended well. A male baby with weight of 2,080 $\mathrm{g}$ was delivered four minutes after the incision. He was transferred to neonatal intensive care unit immediately. One-minute and 5-minute Apgar scores were 8 and 9 . Total duration of surgery was one hour and the

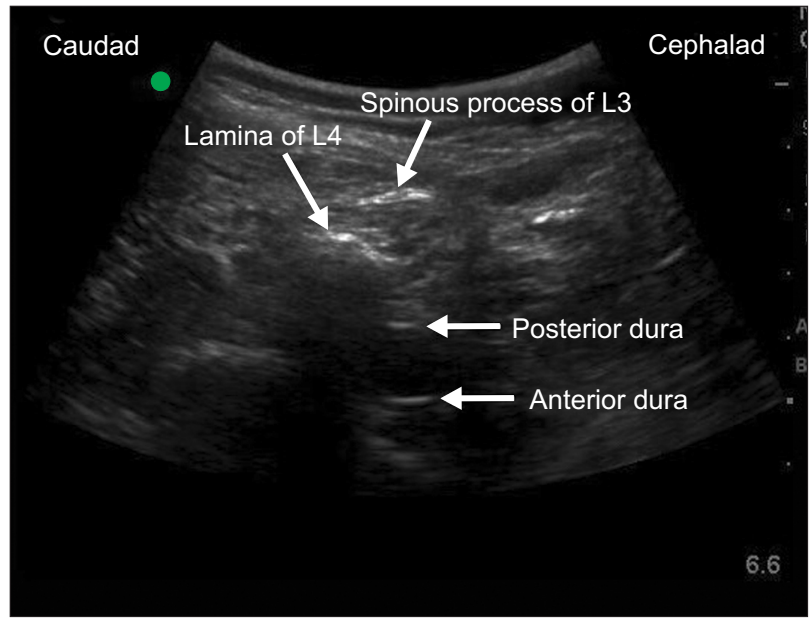

Fig. 2. Ultrasound image immediately real-time ultrasound-guided spinal anesthesia.
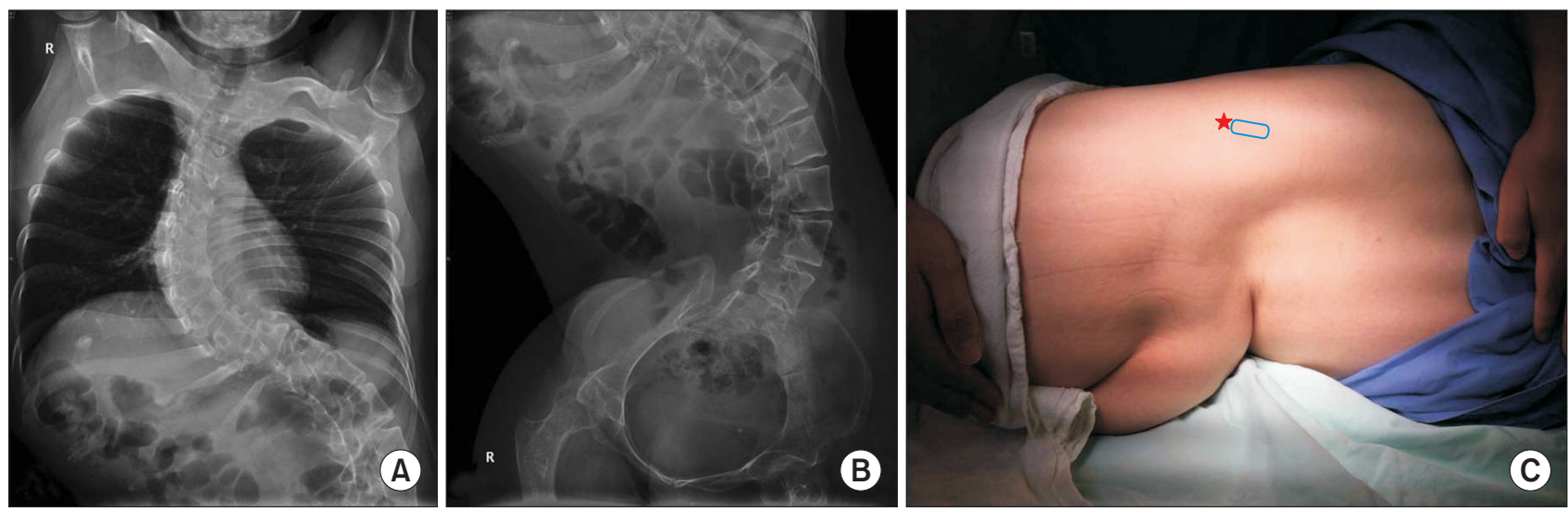

Fig. 1. Chest X-ray (A) and abdomen (B) images before pregnancy. Marked kyphoscoliosis is seen. The patient's surface anatomy and position for real-time ultrasound-guided spinal anesthesia $(C)$. $\downarrow$ : insertion site of the needle, Square: ultrasound probe. 
duration of anesthesia was one hour and 35 minutes. There was no complication related to the spinal anesthesia.

\section{DISCUSSION}

The goal for anesthesia of cesarean section is to provide a safe and adequate anesthesia for mother and the neonate. Regional anesthesia is known to have 16 times less risk, than general anesthesia, since complications such as intubation failure, aspiration of gastric content, and fetal distress due to anesthetics, can be prevented [1].

Therefore, regional anesthesia is recommended for cesarean section. According to American Hospital Association, frequency of regional anesthesia has increased substantially since 1992, compared to general anesthesia that has been decreasing continuously [7].

Single shot spinal anesthesia is the most commonly used regional anesthesia for cesarean section. Absolute contraindications for spinal anesthesia include increase in bleeding tendency due to abnormality in blood coagulation system, infection at the site of injection, and refusal by the patient. In patients without absolute contraindication, spinal anesthesia is avoided when procedural difficulty is increased by severe spinal deformity.

The patient in this case had Duchenne's muscular dystrophy. In the literature, there exist many cases of complications, such as acute rhabdomyolysis, hyperkalemic cardiac arrest, respiratory failure and prolonged mechanical ventilation, due to general anesthesia in Duchenne's muscular dystrophy patients [8]. However, spinal anesthesia was expected to be difficult to perform due to severe hyphoscoliosis. Ultrasound is useful in central neuraxial blocks [9-11]. Also, recent development and spread of ultrasound allowed pre-procedural scan of spine structure before performing spinal anesthesia. Therefore, we attempted spinal anesthesia using ultrasound.

In general, a method of performing spinal anesthesia using ultrasound involves drawing a line connecting spinous processes after locating them using palpation. Once the line is drawn, we can use transverse scan or paramedian sagittal oblique scan to confirm the intrathecal space. The same method may be applied in scoliosis patient too. If spinous process is palpable, a line connecting the spinous processes can be drawn if spinous processes are palpable.

If spinous process is indistinct or impalpable, a line con- necting the spinous process can be drawn, while checking them with ultrasound. Due to rotated spine, most of the lines connecting the spinous process become curvy instead of a straight line. While following the line, the transducer can be rotated and angulated in cephalad-to-caudad and lateral-tomedial direction in order to locate interlaminar space using paramedian sagittal oblique scan. The image of intrathecal space should be found. The degree of rotation, which determines the orientation needed for midline needle insertion, is difficult to estimate clinically or from simple inspection of $\mathrm{X}$-ray, and there is currently no technique suitable for immediate operating room use. Ultrasound can enable estimating the depth and location of the epidural and intrathecal spaces.

Since this case involved an emergency cesarean section of a pregnant women, there was no recent X-ray image of lumbar spine and magnetic resonance imaging evaluation could not be conducted. Also, spinous process was not palpable due to lumbar spine being severely rotated and we were able to locate the interlaminar space and intrathecal space near the very edge of the back (Fig. 1C).

Even pre-procedural ultrasound which is not a real-time guidance is beneficial for performing safe and accurate spinal anesthesia compared to the landmark technique [9-11]. However, the limitation of this ultrasound-assisted technique is that, unlike real-time ultrasound-guided technique, the operator remains 'blind' during the actual needle insertion, and may encounter difficulty in directing the needle through the interlaminar space [11]. We were able to successfully conduct spinal anesthesia at first trial since we used real-time ultrasound-guided technique in this case.

There were reports of successful ultrasound-guided spinal anesthesia in scoliosis patients $[12,13]$. However, there was only one report involving real-time [13] and the location of the insertion site of the needle was not very far from the midline. There has not been a report of conducting real-time ultrasound-guided spinal anesthesia wherein the insertion site of the needle is located far from the midline and close to the flank area due to severe rotated spine.

In conclusion, ultrasound may be beneficial for performing spinal anesthesia in patients with severe spinal deformities that have been difficult to perform spinal anesthesia in the past. 


\section{SUPPLEMENTARY MATERIALS}

Supplementary data is available at https://doi.org/10. 17085/apm.2018.13.4.405.

\section{REFERENCES}

1. Hawkins JL, Koonin LM, Palmer SK, Gibbs CP. Anesthesia-related deaths during obstetric delivery in the United States, 1979 1990. Anesthesiology 1997; 86: 277-84.

2. Carvalho JC. Ultrasound-facilitated epidurals and spinals in obstetrics. Anesthesiol Clin 2008; 26: 145-58.

3. Costello JF, Balki M. Cesarean delivery under ultrasound-guided spinal anesthesia [corrected] in a parturient with poliomyelitis and Harrington instrumentation. Can J Anaesth 2008; 55: 606-11.

4. Prasad GA, Tumber PS, Lupu CM. Ultrasound guided spinal anesthesia. Can J Anaesth 2008; 55: 716-7.

5. Yamaguchi M, Honma E, Mimura M, Yamamoto H, Takahashi E, Namiki A. Identification of the lumbar intervertebral level using ultrasound imaging in a post-laminectomy patient. J Anesth 2006; 20 : 231-3.

6. Grau T, Leipold RW, Conradi R, Martin E. Ultrasound control for presumed difficult epidural puncture. Acta Anaesthesiol Scand 2001; 45: 766-71.

7. Hawkins JL, Gibbs CP, Orleans M, Martin-Salvaj G, Beaty B. Obstetric anesthesia work force survey, 1981 versus 1992. Anesthesiology 1997; 87: 135-43.

8. Hayes J, Veyckemans F, Bissonnette B. Duchenne muscular dystrophy: an old anesthesia problem revisited. Paediatr Anaesth 2008; 18: 100-6.

9. Perlas A. Evidence for the use of ultrasound in neuraxial blocks. Reg Anesth Pain Med 2010; 35: S43-6.

10. Elgueta MF, Duong S, Finlayson RJ, Tran DQ. Ultrasonography for neuraxial blocks: a review of the evidence. Minerva Anestesiol 2017; 83: 512-23.

11. Perlas A, Chaparro LE, Chin KJ. Lumbar neuraxial ultrasound for spinal and epidural anesthesia: a systematic review and metaanalysis. Reg Anesth Pain Med 2016; 41: 251-60.

12. McLeod A, Roche A, Fennelly M. Case series: ultrasonography may assist epidural insertion in scoliosis patients. Can J Anaesth 2005; 52: 717-20.

13. Chin KJ, Chan VW, Ramlogan R, Perlas A. Real-time ultrasoundguided spinal anesthesia in patients with a challenging spinal anatomy: two case reports. Acta Anaesthesiol Scand 2010; 54: $252-5$. 